\title{
Using Ex-Situ TEM to Understand Silica-Supported Ruthenium Catalysts
}

\author{
C.E. Kliewer, S.L. Soled, and S. Miseo
}

ExxonMobil Research and Engineering Co., 1545 Route 22 East, Annandale, NJ 08801

Due to its nonacidic nature, silica remains a potentially interesting support for catalysis. Unfortunately, silica typically has a weak interaction with metal precursors, and thus, generally results in an inhomogeneous (metal particle clustering) catalyst with low dispersion (large metal particles). Studies, however, suggest that the use of organic precursors can mitigate these issues.1-7 Thus, ex-situ TEM work was conducted to better understand this phenomenon.

Chemisorption data were collected on three $0.5 \mathrm{wt} . \% \mathrm{Ru} / \mathrm{SiO}_{2}$ catalysts (Fig. 1). Each catalyst was prepared by a different synthesis route: (1) an aqueous-based synthesis with no organic precursor, (2) an organic precursor-based synthesis followed by a high temperature calcination step, and (3) an organic precursor-based synthesis followed by a low temperature calcination step. Chemisorption reveals that the aqueous-based synthesis initially results in a highly dispersed catalyst but that this catalyst loses surface area fairly quickly upon thermal treatment under hydrogen. An ex-situ TEM study was designed to help explain this behavior. Small, highly clustered Ru particles were found in the aqueous-based preparation (Fig. 2a). The TEM findings are consistent with the aqueous preparation's high initial chemisorption value. A view of the aqueous preparation after a $9 \mathrm{~h}$ hydrogen treatment at $400{ }^{\circ} \mathrm{C}$ shows the same region of catalyst reveals larger metal particles. This TEM data is consistent with the chemisorption data and suggests relatively easy sintering of the small metal particles due to clustering effects (Fig. 2b).

Chemisorption data also indicates that the organic precursor-based synthesis followed by the high temperature calcination resulted in larger but more stable metal particles (Fig. 1). A TEM analysis of the catalyst, revealed larger, randomly-distributed metal particles on the support surface, a result consistent with the chemisorption finding. Thus, through a combination of chemisorption and TEM studies, it is possible to explain the impact of various synthesis protocols on the development of a thermally stable catalyst with good dispersion.

References:

1. S.L. Soled et. al. "A New Approach to Obtain Highly-Dispersed Supported Ruthenium Catalysts" AIChE National Meeting, November 2004.

2. S.L. Soled et. al. "A New Approach to Obtain Highly-Dispersed Supported Ruthenium Catalysts" ACS National Meeting, January 2005.

3. S.L. Soled et. al. "A New Approach to Obtain Highly-Dispersed Supported Ruthenium Catalysts" North American Catalysis Society, May 2005.

4. S.L. Soled et. al. "A New Approach to Obtain Highly-Dispersed, Supported Ru/SiO2 Catalyst" ACS Symposium on Colloidal Surface Science, June 2005.

5. S.L. Soled et. al. "Supported Metal Catalysts: Some Interesting New Leads in an Old Field" 9th International Symposium on the Scientific Basis for the Preparation of Heterogeneous Catalysts, September 2006.

6. S.L. Soled et. al. "Supported Metal Catalysts: Some Interesting New Leads in an Old Field" Studies in Surface Science and Catalysts, 8th International Conference on the Scientific Basis of Preparation of Catalysts, Special Edition - Meeting Proceedings (2005) 1-8.

7. S.L. Soled et. al. "Obtaining Highly-Dispersed, Uniformly Distributed Noble Metal Catalysts on Silica" ACS, March 2012. 


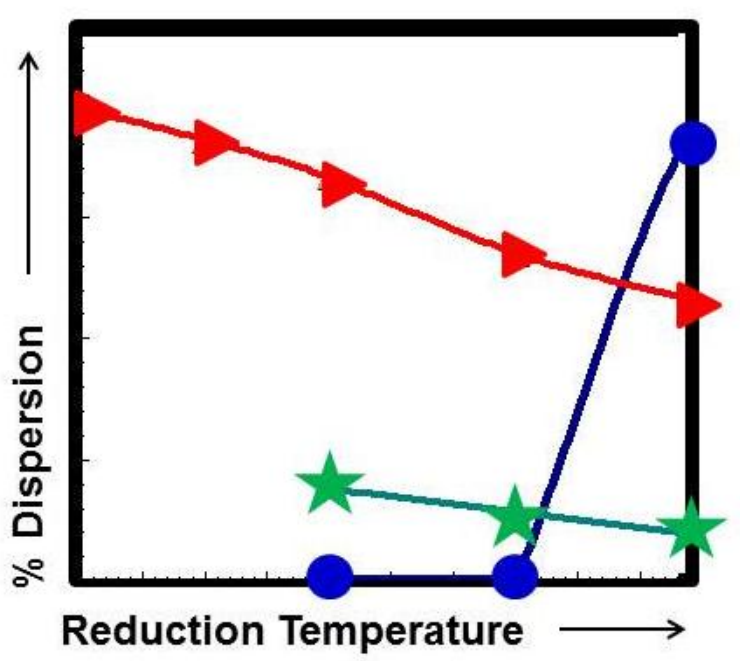

aqueous preparation

organic preparation

followed by high

temperature calcination

organic preparation

followed by low

temperature calcination

Figure 1: Plot reflects changes in chemisorption value for three thermally threated $\mathrm{Ru} / \mathrm{SiO}{ }_{2}$ catalysts prepared by different synthesis routes. The aqueous preparation results in the highest initial dispersion but a significant decrease in dispersion with thermal treatment. The organic preparation followed by a high temperature calcination presents a lower dispersion but remains relatively stable with thermal treatment. A similar organic preparation followed by a low temperature calcination results in a catalyst having the highest dispersion subsequent to the thermal treatment.

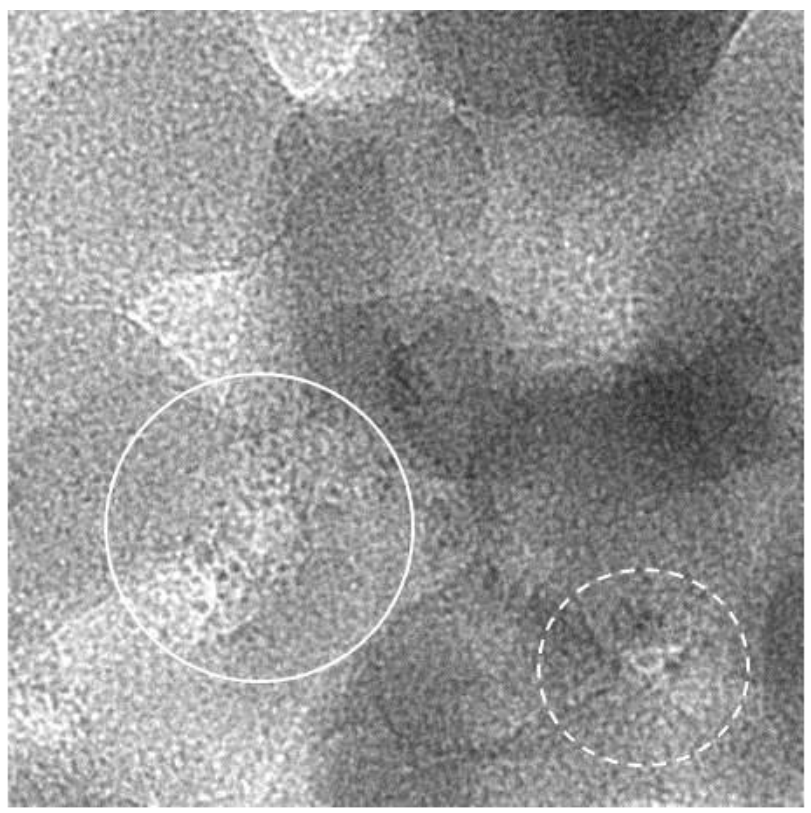

$\mathrm{R}: 150^{\circ} \mathrm{C}, 3 \mathrm{hr}$.

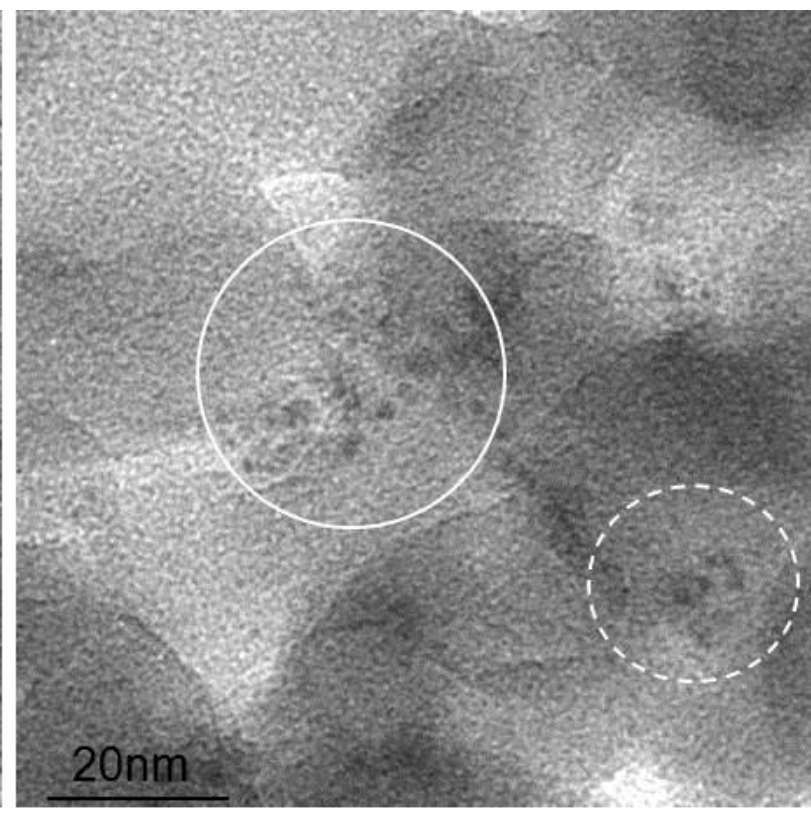

$\mathrm{R}: 150^{\circ} \mathrm{C}, 3 \mathrm{hr}$.

$\mathrm{R}: 400^{\circ} \mathrm{C}, 9 \mathrm{hr}$.

Figure 2: Bright field TEM image presents (a) morphology of $\mathrm{Ru} / \mathrm{SiO}_{2}$ catalyst prepared via aqueous-based synthesis protocol after $150{ }^{\circ} \mathrm{C}$ reduction for $3 \mathrm{~h}$ and (b) the same area after an additional $400{ }^{\circ} \mathrm{C}$ reduction for $9 \mathrm{~h}$. Small metal particle clustering leads to sintering during the thermal treatment and results in the decreased chemisorption value. 